\title{
Cluster based Energy Efficient Routing Protocol (EERP) for Mobile Wireless Sensor Network
}

\author{
Atul Garg, Kavita Gupta, Aarti Singh
}

\begin{abstract}
Energy efficiency is one of the major open research challenges for Mobile Wireless Sensor Networks. This paper evaluates EERP which is an integrated solution for routing data according to user preferences and context. Proposed protocol contains layers for optimal cluster head election, data aggregation and filtering of sensed data. Each layer deploys intelligent mobile agents for performing its designated function efficiently. The work has been evaluated on standard metrics available in literature. Initial implementation and evaluation indicated extended network life time, transmission delay and higher packet delivery ratio as well.
\end{abstract}

Keywords : MWSN, Routing, Energy Efficiency, Mobile Agents, Data Filtering.

\section{INTRODUCTION}

Mobile Wireless Sensor Network (MWSN) is the arrangement of small tiny battery operated sensor nodes. The field of MWSN came into existence to overcome the issues of static wireless network like energy consumption rate of the close nodes, scarce battery life, routing overhead etc. Each node is capable to perform various tasks like sensing of data, communication in the network and transmission of data to the base station. Grilling of literature [1,2] reveals that routing protocols performance depends upon various factors. In the literature many researchers $[3,4,5]$ have proposed diversity of protocols which are suitable for MWSN; from these proposed protocols very few of them gave desirable output but, to some extent also the overall performance of protocol was not appreciable. As it is already stated lifetime of network and residual energy of nodes has a directly relationship [4].Lifetime of the network can be extended, if the energy of sensor network is consumed in best possible way. The remaining time of stay of nodes is calculated in terms of their total energy, and also the energy consumed per unit of time.

The work begins with emphasizing the problem pertaining to routing in MWSN, the proposed solution and later it explores parameters suitable for evaluation of the proposed algorithms [14]. MWSN is desired to be energy efficient and

Revised Manuscript Received on October 15, 2019.

* Correspondence Author

Atul Garg ${ }^{1}$, Kavita Gupta ${ }^{2}$, Aarti Singh ${ }^{3}$

${ }^{1}$ Chitkara University Institute of Engineering and Technology, Chitkara University, Punjab, India,

${ }^{2}$ Research Scholar, M.M. Institute of Computer Technology \& Business Management, Maharishi Markandeshwar (Deemed to be University), Mullana, Haryana, India India

${ }^{3}$ Assistant Professor, Guru Nanak Girls College, Yamuna Nagar, Haryana, long-lasting network life time. Here efficiency of network is defined in terms of residual energy of nodes and their remaining time of stay in network. This research work is structured as follows: Section 2 highlight the work of renowned researchers, Section 3 presents brief introduction about the Energy Efficient Routing Protocol (EERP) and explores the various parameters for evaluation of MWSNs which may be used for evaluating performance of proposed work. Section 4 is devoted to implementation details, results and discussion. Section 5 finally concludes this work.

\section{RELATED WORK}

This work emphasis on sustaining energy level of sensor nodes in order to analyse the performance of EERP. The detailed study of existing works reveals that clustering proves to be the best solution for prolonged network lifetime. However; there are numerous approaches existing for WSN but all doesn't work efficiently for MWSN. This work is motivated by the gaps pertaining to fitness of clustering in MWSN. The primary understanding of this field recommended the consideration of M-LEACH [6] protocol for cluster formation which shown to be a successful attempt. However; simulation result of M-LEACH proved to be insufficient for cluster based mobile network (shown later in results). Awwad et.al proposed a Cluster Based Routing (CBR) [7] approach assumed to offer an efficient network but issue related to cluster head was still prevailing. PEGASIS was also assumed to be suitable protocol for sensor networks but it was not an energy efficient approach [8]. None of these existing approaches used intelligent agents to accomplish clustering process. In existing [7] approach, probabilistic function was used to elect Cluster Head $(\mathrm{CH})$ for each simulation round and data cluster head transmitted to base station. But mobile nature of sensor nodes also causes the movement of $\mathrm{CH}$ thereby enduring the cluster unattended till the complete simulation round. Therefore, there was need of an optimal and efficient cluster head election protocol, which motivates the proposal of an optimal cluster head election protocol.

Researchers have proposed various approaches $[5,7,8]$ which have been used for comparing the performance of EERP, but among all those approaches CBR and M-LEACH were the comparable clustered based approaches. Transmission delay, energy consumption rate, packets delivery ratio, network life time are assumed to be the suitable parameters for analysing the performance of a routing protocol [9]. Performance of EERP is evaluated using above mentioned parameters. The next section outlines the layer wise working of the proposed approach. 


\section{ENERGY EFFICIENT ROUTING PROTOCOL : AN OVERVIEW}

The Energy Efficient Routing Protocol (EERP) is a clustering based routing protocol that considers conserving energy consumption rate to its optimal level at every step of communication. This protocol exploits basic M-LEACH protocol for cluster formation. Routing protocol stack in MWSN mainly consists of Cluster Head Election Layer, Data Aggregation Layer, Data Filtration Layer. Each layer is responsible to perform different types of tasks. While each layer in EERP is autonomous but it also works in coordination with other layers. Following subsections illustrates the working of the mentioned layers.

\section{- Cluster Head Election Layer}

Cluster Head Election layer is responsible for electing the cluster head among the participating nodes in the network. Sensor nodes in sensor networks have self-organizing capability and main objective is to create infrastructure for network. Sensor nodes deployment and cluster head election task is accomplished at physical layer. Literature $[2,6,8,10$, $11,12]$ reveals that clustering is one of the key solution for MWSN. This layer elects one of the participating mobile sensors as cluster head on basis of amount of available energy, remaining time of stay in the network and distance of respective node from the base station $[14,15]$.

\section{- Data Aggregation Layer}

This layer contains the mechanism for data aggregation that is accomplished with help of mobile agents. Each level of hierarchical network has been assigned mobile agent that collects the data from its member nodes so as to remove redundancy from the sensed data [16]. Since the nodes lie in close neighbourhood in a sensor network, thus there is high probability of neighbouring nodes sensing similar data. Mobile agent removes redundant data and sends the fresh aggregated copy of data to the next high level.

\section{- Data Filtering Layer}

The main task performed at this layer is filtering of the aggregated data received from its immediately lower layer. Filtering agents are being used to perform filtering of the data so as to segregate noise from the collected data. Proposed filtering mechanism executes the filtering process in two ways (1) Demand Filtering (2) Periodic Filtering. In the former approach data filtering is accomplished whenever demand for data filtering is received at this layer, whereas in later approach data filtering task gets executed on demand basis [13].

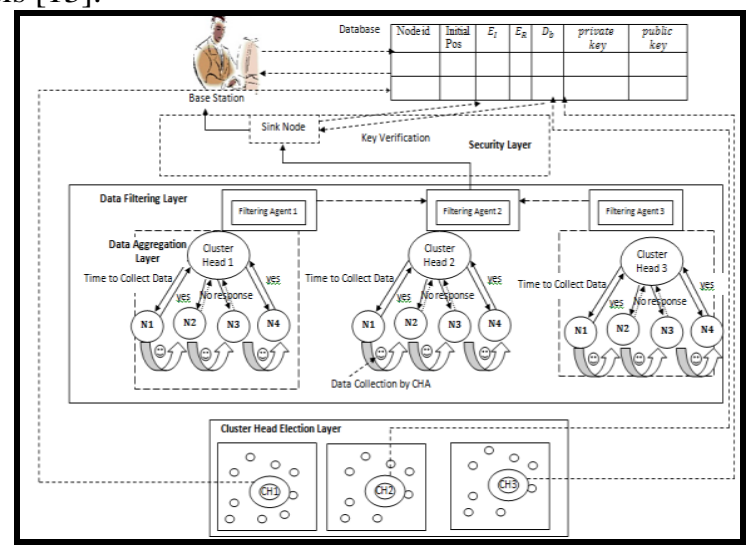

Figure 1: Detailed view of Energy Efficient Routing Protocol (EERP)

\begin{tabular}{ll}
$\odot$ & Cluster Head Agent \\
\hline$\square$ & Filtering Agent \\
\hline$E_{I}$ & Initial Energy \\
$E_{R}$ & Residual Energy \\
$D_{b}$ & Distance from base station \\
$\mathrm{CH}$ & Cluster Head
\end{tabular}

Upcoming section highlights the performance of Energy Efficient Routing Protocol (EERP), CBR, M-LEACH.

\section{IMPLEMENTATION DETAILS}

All three modules (Cluster Head Election, Data Aggregation, Data filtration) of EERP have been implemented in MATLAB (2015a), as MATLAB is a better simulator for mobile nodes. The proposed approach is implemented for a scenario of 100 sensor nodes. All nodes are divided into clusters on the basis of the distance among them. The algorithm [14] for election of cluster head is simulated in the network of $100 \mathrm{~m} \times 100 \mathrm{~m}$. At initial state all nodes were having fixed energy $50 \mathrm{~J}$ and also all nodes assumed to consume a fix amount of energy i.e. 10J for receiving and transmission of the data packets over the network. The nodes while moving or in idle state are assumed to consume $5 \mathrm{~J}$ of energy respectively. During the implementation it is observed that energy of few nodes got depleted to zero and such nodes are known as dead nodes.

\section{RESULTS ANALYSIS OF EERP}

It is already stated above that there are conspicuous factors measuring the performance of EERP are network life time (Residual Energy), delay of packet transmission and packet delivery ratio. Therefore, the performance of EERP is evaluated on above mentioned parameters.

\section{A.}

\section{Network Life Time}

As it is already stated above sensor nodes are battery operated, therefore lifetime depends upon the amount of available energy. Energy (of nodes) consumed in various ways: during communication (receiving and transmission of data packets), energy consumed during the movement of mobile nodes and in an idle state. It can be observed that in case of uncompromised network, EERP has the highest energy level followed by CBR and M-LEACH has the minimum energy level. In case of single intruder, it slightly declines for EERP but CBR and M-LEACH could not manage their energy levels. If malicious node density reaches up to 5 to 20 nodes, in that case, for all protocols, energy level is drained out. Further, Figure 2 represents the rate of depreciation of energy and available energy of nodes for the network (noted for 8000 rounds). Results obtained reflect that higher residual energy level offers energy efficient network as per desired objective. Additionally, now the newly elected $\mathrm{CH}$ has ample energy and elongated stay time in the network. Thus, $\mathrm{CH}$ is required to be performed less times. 
Thereby, saving energy and increasing network lifetime. It has been observed that network lifetime increased approximately by $25 \%-30 \%$ with this proposed EERP as compared to traditional approaches.

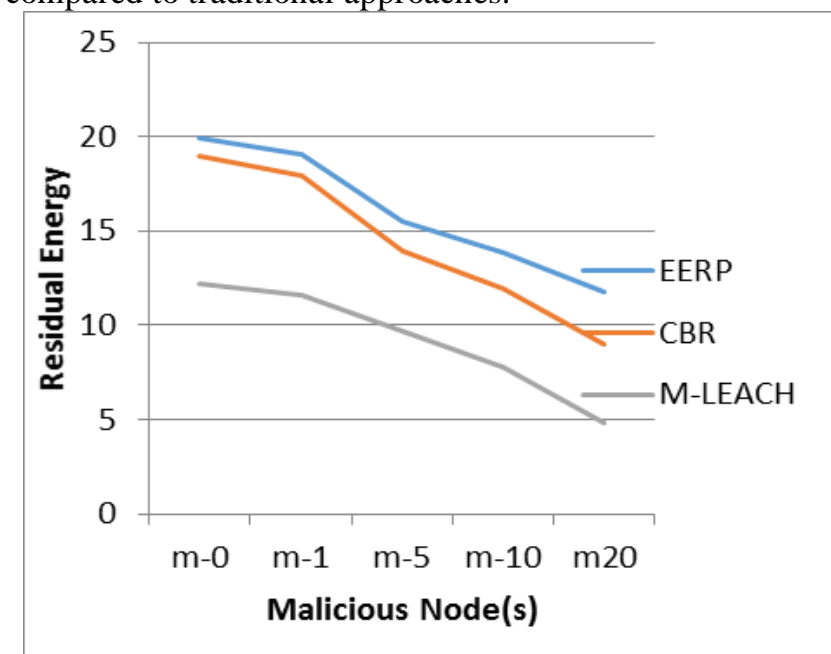

\section{B. Transmission Delay}

Figure 2: Residual Energy

As per Figure 3, in case of uncompromised network, EERP has the minimum delay followed by CBR and M-LEACH has the highest Delay. In case of single intruder, it slightly increases but after that it varies w.r.t. the malicious node's density. In case of malicious node density 20 , it reaches up to its peak level for each protocol and it can be observed that M-LEACH has the highest Delay followed by CBR whereas it is minimum for EERP. However, for EERP and CBR, it is gradually increasing.

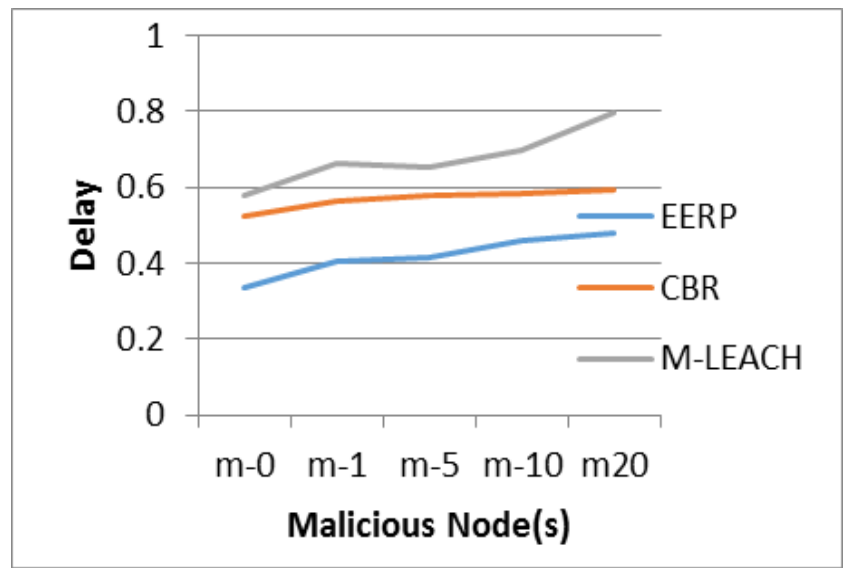

Figure 3: Transmission Delay of EERP vs. CBR vs. M-LEACH

\section{C.Packet Delivery Ratio}

In sensor network, Packet Delivery Ratio (PDR) is one of the significant parameter. It is defined in terms of number of packets received and dropped, high value of PDR ensures to offer efficient network. Figure 4 depicts the ratio, number of packets transmitted in the network. For PDR estimation both

number of packets received and transmitted takes in consideration for each simulation round individually. As it is already stated PDR and number of packets received has an inversely proportional. In proposed approach EERP, use of Mobile Agents at various levels of cluster based protocol

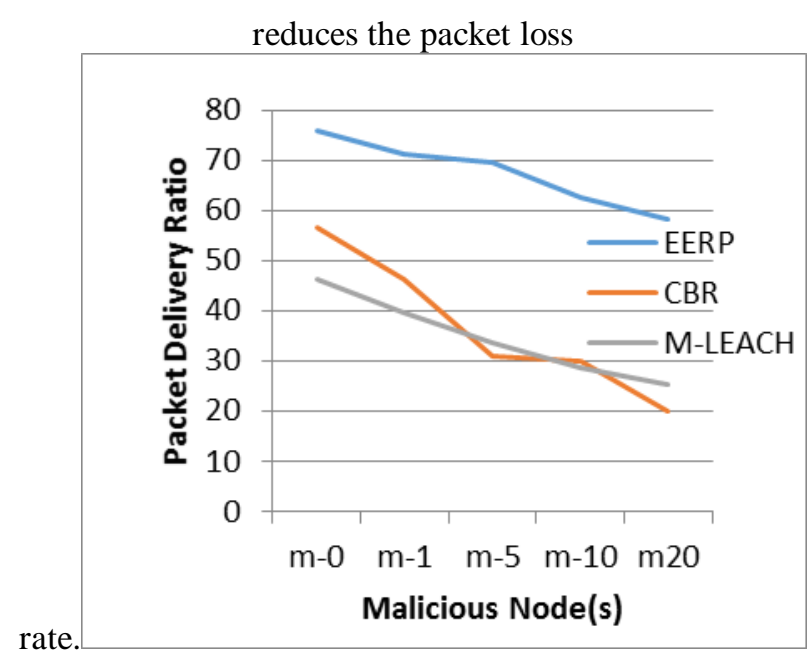

Figure 4: Packet Delivery Ratio-EERP vs. CBR vs.

\section{M-LEACH}

\section{CONCLUSION}

In this research work, a multi-layered agent based clustering scheme for MWSN is proposed. Use of Mobile Agents (MA) at different layers improves the performance of the protocol. Mobile Agents are cheaper and the routing cost is not affected with multiple use of MA. Performance of proposed approach is evaluated on the basis of transmission delay, packet delivery ratio, network lifetime. For simulation purposes of proposed EERP, MATLAB platform is used. Implementation results obtained are promising in terms of extended network lifetime. Further agent based data filtering and aggregation scheme improves the packet delivery ratio. In future, a security provision will be embedded with EERP, in order to guard the network against replication attack.

\section{References}

1. Akyildiz I.F., et al.,"Wireless sensor networks: a survey. Computer Networks", 38: 393-422 (2002)

2. Anitha, R.U., Kamalakkannan P., "Energy efficient luster head selection algorithm in mobile wireless sensor networks", International Conference on Computer Communication and Informatics (ICCCI). 1-5 (2003).

3. Almazaydeh L., Abdelfattah E., Al-Bzoor M., Al-Rahayfeh, A. "Performance evaluation of routing protocols in wireless sensor networks", International Journal of Computer Science and Information Technology, 2(2):64-73(2010).

4. Choi L., JungJ.K.,ChoB.-H., Choi H.,"M-Geocast: robust and energy efficient geometric routing for mobile sensor networks", International federation for information processing (SEUS), 5287: 304-316 (2008).

5. Akkaya K.,Younis M.,"A survey on routing protocols for wireless sensor networks”, AdHoc Networks, 3: 325-329(2005).

6. Saini P., Sharma A.K.,"Energy Efficient Scheme for Clustering Protocol Prolonging the

Lifetime of Heterogeneous Wireless Sensor Networks", International Journal of Computer Applications, 6(2) (2012).

7. A. B. Awwad, Chee K. Ng, Nor K. Noordin, and Mohd. Fadlee A Rasid.,"Cluster Based Routing Protocol for Mobile Nodes in Wireless Sensor Network", IEEE transactions: 233-241(2009).

8. Tri Gia Nguyen, So-InC.,"Two energy-efficient cluster head selection techniques based on distance for wireless sensor networks", International conference on Computer Science \& Engineering, 33-38 (2014).

9. AL-Kraki J., E.KamalA.,"Routing Techniques In Wireless Sensor network- A survey", IEEE Communications. (2004).

10. Bhat V., Shenoy S.U. Effective Cluster Head Selection Based on EDM for WSN. The IUP Journal of Computer Sciences, 4: 9-13 (2014). 
11.Gupta D., VermaR.,"An enhanced cluster-head selection scheme for distributed heterogeneous wireless sensor network", International Conference on Advances in Computing, Communications and Informatics (ICACCI). 1684 - 1689 (2014).

12.MadaniS.A.,"Clustering-based power-controlled routing for mobile wireless sensor networks", International Journal of Communication Systems.: 25(4): 529-542 (2012).

13. Singh A., Gupta K.,"Hybrid Filtering Approach for Data Filtering in Mobile Wireless Sensor Networks", International Journal of Computer Applications, 138(8): 37-41(2016).

14.Singh A., Gupta K., "Optimal Cluster Head Election Algorithm for Mobile Wireless Sensor Networks", Published in proceedings of ICTCS in ACM. (2016).

15.Singh A., Gupta K., Singh R., Mukherjee S., "An Improved Cluster Head Selection Algorithm for Mobile Wireless Sensor Network", Journal of Network Communication and Emerging Technology, 5(2) : 170-174 (2015).

16.Juneja D., Singh A., Gupta K.,"Exploiting Mobility of Agents for Data Sharing and Aggregation in a Clustered Mobile Wireless Sensor networks", Journal of Network Communication and Emerging Technology. 5(2): 118-124 (2015). 